\title{
Synchrotron radiation in odd dimensions
}

\author{
D. V. Gal'tsov" and M. Khlopunov $\odot^{\dagger}$ \\ Faculty of Physics, Moscow State University, 119899 Moscow, Russia
}

(Received 9 March 2020; accepted 9 April 2020; published 24 April 2020)

\begin{abstract}
In odd space-time dimensions, the retarded solution of the massless wave equation has support not only on the light cone, but also inside it. At the same time, a free massless field should propagate at the speed of light. The mismatch of these two features is resolved by the fact that the emitted part of the field in the wave zone depends on the history of motion up to the retarded moment of proper time. It is shown that in the case of circular motion with an ultrarelativistic velocity, the main contribution to the radiation amplitude is made by a small interval of proper time preceding the retarded time, and thus the tail term is effectively localized. We obtain a tentative formula for scalar synchrotron radiation in $D$ dimensions: $P=g^{2}\left(\omega_{0} \gamma^{2} / \sqrt{3}\right)^{D-2}$, which is explicitly verified in $D=3,4,5$.
\end{abstract}

DOI: $10.1103 /$ PhysRevD.101.084054

\section{INTRODUCTION}

Over the past two decades, interest has arisen in the theory of radiation in space-time dimensions other than four. This was due to the emergence of theories with large extra dimensions [1], the development of a holographic approach to the description of quark-gluon plasma [2,3], the beginning of gravitational-wave astronomy, and other reasons. The case of three-dimensional space-time became relevant in connection with the development of fieldtheoretical models in condensed matter physics, such as the quantum Hall effect, high-temperature superconductivity, and graphene. Radiation is the main classical and quantum process of the interaction of charged particles with massless fields, which are basic ingredients of a number of theories. Therefore, it seems to be of primary importance to study the general laws of radiation in space-times other than four-dimensional.

More than a hundred years ago, Ehrenfest [4] called the features of radiation in four-dimensional electrodynamics a unique manifestation of the dimensionality of spacetime, so different are the physical effects and the theoretical description of radiation in other dimensions. General features of wave propagation in arbitrary dimensions were discussed in the classical collections of mathematical physics, such as Courant and Hilbert [5], lectures of Hadamard [6], the book by Ivanenko and Sokolov [7].

\footnotetext{
*galtsov@phys.msu.ru

"khlopunov.mi14@physics.msu.ru
}

Published by the American Physical Society under the terms of the Creative Commons Attribution 4.0 International license. Further distribution of this work must maintain attribution to the author(s) and the published article's title, journal citation, and DOI. Funded by SCOAP .
It was found that there is a fundamental difference between even and odd dimensions consisting in the failure of the Huygens principle in the odd case. In odd-dimensional space-times, the signal from an instantaneous flash of current reaches the observer through the time interval necessary for the signal to propagate at the speed of light, but then the tail is observed endlessly. In even-dimensional spaces, this is not so: the instantaneous signal ends instantly at the observation point. The mathematical reason is that the retarded Green's function of the d'Alembert equation in odd dimensions has a support localized not only on the light cone, but also inside it.

There is an intriguing opportunity to discover an additional dimension in the five-dimensional theory of RandalSundrum and other similar theories by scanning it with light signals [8]. The idea of a direct experimental search for additional dimensions was discussed in anticipation of the future development of the LISA [9]. The propagation of gravitational waves in the presence of additional dimensions and the possibility of observational effects was discussed recently in Ref. [10]. The birth of gravitational-wave astronomy has given a new impetus to the search for opportunities to explore the dimensionality of space-time. According to some theories, gravitational waves will leak into extra dimensions, causing attenuation of waves during propagation in the Universe. Although such an effect was not detected in the event of the fusion of neutron stars GW170817 [11], the possibility of using the astronomical data of several messengers to limit the curvature of additional dimensions is not ruled out [12], further studies continue, see recent articles and links therein [13-15]. We should also mention the connection between the extra dimensions and the black hole shadows [16].

Motivation for studying radiation in various dimensions comes also from holography, namely, the holographic 
modeling of quark-gluon plasma [2]. From the gravity side it involves consideration of gravitational radiation in collisions of ultrarelativistic particles or approximating them shock gravitational waves on the background of higherdimensional anti-de Sitter [3]. Radiation in higher dimensions is relevant to the problem of creation of black holes in particle collisions in theories with large extra dimensions [17-19]. Note also an interesting interplay of even- and odddimensional features encountered in the black hole brane [20] and the particle brane [21,22] systems.

As was said, the behavior of massless fields and radiation features in even and odd dimensions is rather different, to be more familiar in the even case. In most of the existing literature the even-dimensional problems were considered [18,23-27], while the case of odd dimensions was discussed only in the context of the radiation reaction problem [28-36], see also the reviews [37,38]. In four [39] and higher even dimensions, the subtraction of infinite terms can be performed following the Gelfand and Shilov distribution theory [40], which leads to a differential equation with higher derivatives, that resembles the "rigid particle" dynamics [41-43]. In odd dimensions, there is a nonlocal tail term, as in the case of four-dimensional curved space, as was discovered by DeWitt and Brehme [44] (for a recent review see Ref. [45]). Tail in curved space-time is due to the scattering of waves by the curvature of spacetime and its evaluation is rather complicated. In contrast, the tail terms in odd dimensions are analytically known in the closed form and they can be understood in terms of dimensional reduction (see below). Note that in higher even-dimensional curved space-times additional geometrical terms appear [46].

As for genuine radiation processes, with the exception of the problem of high-energy bremsstrahlung, insensitive to whether the number of dimensions is even or odd [17,19], and a brief note [47], where the use of fractional derivatives for the Green's functions was demonstrated, as far as we know, this article is the first attempt to calculate radiation in odd dimensions using the standard wave zone approach [48], including an explicit calculation of synchrotron radiation in three and five dimensions. We use the Rohrlich definition of radiation $[49,50]$ and its refinement due to Teitelboim [51] (see also Refs. [23,52,53]) to prove that the long-range component of the retarded potentials corresponds to radiation in the same way as in the four dimensions. This component, however, depends on the history of the particle motion preceding the retarded time, but not only at the retarded time, as in even-dimensional space-time. Our results were double checked by calculating the spectral distribution of radiation, which is insensitive to whether the dimension is even or odd. We expect they can be tested also within the framework of an effective field theory approach developed in Refs. [54-56].

The paper is organized as follows. In the second section, we briefly recall the derivation of the recurrent relations between odd-dimensional Green's functions of d'Alembert equation by Ivanenko and Sokolov. We discuss the regularization of the generalized functions involved following the Gelfand and Shilov's formalism and explain our method of regularization considering them as the product of two distributions. Section III is devoted to the calculation of scalar synchrotron radiation in $(2+1)$-dimensional spacetime using both the coordinate representation for the retarded Green's function in the wave zone and the Fourier spectral decomposition. In Sec. IV, we perform similar calculations in $(4+1)$-dimensional space-time. We find a universal formula for synchrotron radiation verified in $D=3,4,5$ and supposedly valid in any $D$. In the last section we briefly formulate our results and discuss relation to other work.

\section{THE SETUP}

We write the action of the massive relativistic particle interacting with the massless scalar field in $D=n+1$ dimensional Minkowski space-time as

$$
\begin{aligned}
S= & -\int(m+g \varphi(z)) \sqrt{\eta_{\alpha \beta} \dot{z}^{\alpha} \dot{z}^{\beta}} d \tau \\
& +\frac{1}{2 \Omega} \int \eta^{\mu \nu} \partial_{\mu} \varphi(x) \partial_{\nu} \varphi(x) d^{n+1} x,
\end{aligned}
$$

where $m$ is the particle's mass, $g$ is the scalar charge, $z^{\mu}(\tau)$ is the particle's worldline, $\dot{z}^{\mu}(\tau)=d z^{\mu}(\tau) / d \tau$. Here $\Omega$ is the area of the $(n-1)$-dimensional sphere of unit radius:

$$
\Omega=\frac{2 \pi^{n / 2}}{\Gamma(n / 2)}
$$

The Minkowski metric is $\eta_{\mu \nu}=\operatorname{diag}(1,-1, \ldots,-1)$. Our choice of the scalar coupling constant $g$ differs from a more frequent definition $f=g / m$ (see, e.g., Ref. [57]) in a way to make it nonzero in the massless limit $m \rightarrow 0$.

This action leads to the following wave equation for the scalar field

$$
\begin{gathered}
\square \varphi(x)=-\Omega j(x), \\
j(x)=g \int d \tau\left(\dot{z}^{\alpha} \dot{z}_{\alpha}\right)^{1 / 2} \delta^{n+1}(x-z(\tau)),
\end{gathered}
$$

where $j(x)$ is the current and $\square=\partial^{\mu} \partial_{\mu}$ is d'Alembert operator. The canonical energy-momentum tensor of the scalar field is

$$
T_{\mu \nu}(x)=\frac{1}{\Omega}\left(\partial_{\mu} \varphi \partial_{\nu} \varphi-\frac{1}{2} g_{\mu \nu} \partial_{\alpha} \varphi \partial^{\alpha} \varphi\right)
$$




\section{A. Green functions in odd dimensions}

For reader's conveniences, we recall here Ivanenko and Sokolov's derivation of the recurrent relation for odddimensional Green functions of the scalar d'Alembert equation. We present the retarded solution of the Eq. (2.2) as

$$
\begin{gathered}
\varphi(x)=-\Omega \int d^{n+1} x^{\prime} G_{\mathrm{ret}}^{n+1}\left(x-x^{\prime}\right) j\left(x^{\prime}\right), \\
\square G_{\mathrm{ret}}^{n+1}\left(x-x^{\prime}\right)=\delta^{n+1}\left(x-x^{\prime}\right),
\end{gathered}
$$

where the retarded Green's function of the d'Alembert equation is

$$
G_{\mathrm{ret}}^{n+1}\left(x-x^{\prime}\right)=-\int \frac{d^{n+1} k}{(2 \pi)^{n+1}} \frac{e^{-i k\left(x-x^{\prime}\right)}}{k^{2}+i \varepsilon k^{0}},
$$

with $k^{2}=k_{\mu} k^{\mu}$ and $\varepsilon=+0$ defining the correct shift of a pole in the complex plane $k^{0}$. After integration over $k^{0}$, we obtain an integral over the Euclidean $n$-dimensional space:

$$
\begin{array}{r}
G_{\mathrm{ret}}^{n+1}\left(x-x^{\prime}\right)=\int \frac{d^{n} k}{(2 \pi)^{n}} \frac{\sin \omega T}{\omega} e^{i \mathbf{k} \mathbf{R},} \\
\mathbf{R}=\mathbf{r}-\mathbf{r}^{\prime} ; \quad T=x^{0}-x^{\prime 0} ; \quad \omega=|\mathbf{k}| .
\end{array}
$$

One can introduce the hyperspherical coordinates [58] and integrate over the cyclic angles, obtaining

$$
\begin{aligned}
G_{\mathrm{ret}}^{n+1}\left(x-x^{\prime}\right)= & \frac{2 \pi^{(n-1) / 2}}{\Gamma((n-1) / 2)} \int \frac{d \omega d \theta_{n-2}}{(2 \pi)^{n}} \omega^{n-2} \sin (\omega T) \\
& \times \sin ^{n-2}\left(\theta_{n-2}\right) e^{i \omega R \cos \theta_{n-2}}
\end{aligned}
$$

The remaining angular integral can be expressed through the Bessel function of the order $\nu-1$, with $n=2 \nu[59,60]$ :

$$
G_{\text {ret }}^{2 \nu+1}\left(x-x^{\prime}\right)=\frac{R}{(2 \pi R)^{\nu}} \int_{0}^{\infty} d \omega \omega^{\nu-1} J_{\nu-1}(\omega R) \sin \omega T .
$$

Using the recurrent relations between the Bessel functions

$$
\left(\frac{d}{x d x}\right)^{m} \frac{J_{n}(x)}{x^{n}}=(-1)^{m} \frac{J_{n+m}(x)}{x^{n+m}}
$$

we obtain the following generating formula:

$$
G_{\mathrm{ret}}^{2 \nu+1}(X)=\frac{(-1)^{\nu-1}}{(2 \pi)^{\nu-1}} \frac{d^{\nu-1}}{(R d R)^{\nu-1}} G_{\mathrm{ret}}^{2+1}(X) .
$$

For $\nu=1$ one has

$$
G_{\mathrm{ret}}^{2+1}\left(x-x^{\prime}\right)=\frac{1}{2 \pi} \int_{0}^{\infty} d \omega J_{0}(\omega R) \sin \omega T .
$$

This integral gives the Heaviside function $[7,60]$, so we obtain

$$
G_{\mathrm{ret}}^{2+1}(X)=\frac{\theta\left(X^{0}\right)}{2 \pi} \frac{\theta\left(X^{2}\right)}{\sqrt{X^{2}}}
$$

where $X^{\mu}=x^{\mu}-x^{\prime \mu}$. This function is localized inside the future light cone $X^{2}=0$. It can be considered as a $\lambda=-1 / 2$ member of the family of generalized functions $x_{+}^{\lambda}$ [40] defined as a linear continuous functional on the space of test functions $\psi(x)$ infinitely differentiable and zero outside some bounded region. It is defined as

$$
x_{+}^{\lambda}= \begin{cases}x^{\lambda}, & \text { if } x>0, \\ 0, & \text { if } x<0\end{cases}
$$

for any complex $\lambda$ (here we assume $\lambda$ real). For test functions which are zero in a small vicinity of the origin $x=0$ the functional is defined by the usual integration. For $-1<\lambda<0$ the integral is finite for any test $\psi(x)$, so one defines the contraction as

$$
\left(x_{+}^{\lambda}, \psi(x)\right)=\int_{0}^{\infty} x^{\lambda} \psi(x) d x .
$$

Differentiation according to the rule

$$
\frac{d}{d x} x_{+}^{\lambda}=\lambda x_{+}^{\lambda-1}, \quad \lambda \neq-1,-2, \ldots,
$$

increases the degree of singularity, but we can define the regularization differentiating in the sense of distributions,

$$
\left(\left(x_{+}^{\lambda}\right)^{\prime}, \psi(x)\right)=-\left(x_{+}^{\lambda}, \psi^{\prime}(x)\right)=-\lim _{\epsilon \rightarrow 0} \int_{\epsilon}^{\infty} x^{\lambda} \psi^{\prime}(x) d x,
$$

and then integrating by parts with $\psi^{\prime}(x) d x=d u$, $x^{\lambda}=v, u=\psi(x)+C$ to get

$$
\begin{aligned}
\left(\left(x_{+}^{\lambda}\right)^{\prime}, \psi(x)\right)= & -\lim _{\epsilon \rightarrow 0}\left[\left.x^{\lambda}(\psi(x)+C)\right|_{\epsilon} ^{\infty}\right. \\
& \left.-\int_{\epsilon}^{\infty} \lambda x^{\lambda-1}(\psi(x)+C)\right] d x .
\end{aligned}
$$

The substitution becomes zero if one chooses $C=-\psi(0)$, which can be considered as regularization of the infinite integral. Then we obtain the derivative as the regularized functional

$$
\left(\left(x_{+}^{\lambda}\right)^{\prime}, \psi(x)\right)=\int_{0}^{\infty} \lambda x^{\lambda-1}(\psi(x)-\psi(0)) d x,
$$


which actually defines the functional (2.11) for $-2<$ $\lambda<-1$. Similarly, for $-(n+1)<\lambda<-n$, we get the regularized functional as

$$
\begin{aligned}
\left(x_{+}^{\lambda}, \psi(x)\right)= & \int_{0}^{\infty} x^{\lambda}\left(\psi(x)-\psi(0)-x \psi^{\prime}(0)-\cdots\right. \\
& \left.\left.-\frac{x^{n-1}}{(n-1) !} \psi^{(n-1)}(0)\right)\right) d x .
\end{aligned}
$$

For negative integer $\lambda$, a proper definition of the regular functional is the derivative of the delta function [40],

$$
\lim _{\lambda \rightarrow-n} \frac{x_{+}^{\lambda}}{\Gamma(\lambda+1)}=\delta^{(n-1)}(x) .
$$

Using these definitions one can construct the Green's functions as regularized functionals for any dimension.

In practical calculations, however, it is more convenient to present $x_{+}^{\lambda}$ as a singular product of two distributions, as it stands in Eq. (2.10), $x_{+}^{\lambda}=\theta(x) x^{\lambda}$, and differentiate it using the Leibnitz rule

$$
\frac{d}{d x}\left(\theta(x) x^{\lambda}\right)=\delta(x) x^{\lambda}+\lambda x^{\lambda-1} \theta(x) .
$$

For negative $\lambda$ this is the sum of two singular expressions, but if we regularize them by shifting $x \rightarrow x+\epsilon$ in delta and theta functions, the divergences in two terms can be shown to cancel, so the derivative will be a good distribution which can be checked to correspond to the definitions in Ref. [40]. In what follows we will use this second prescription demonstrating cancellation of divergences explicitly.

\section{B. Cancellation of divergences in the static limit}

Let us verify the absence of divergences in the field of a static particle in the case of $(4+1)$ dimensions, which is the most transparent illustration.

From Eq. (2.9), performing differentiation according to Eq. (2.12), we find the retarded $(4+1)$-dimensional Green function as a sum of two terms

$$
G_{\mathrm{ret}}^{4+1}(X)=\frac{\theta\left(X^{0}\right)}{2 \pi^{2}}\left(\frac{\delta\left(X^{2}\right)}{\left(X^{2}\right)^{1 / 2}}-\frac{1}{2} \frac{\theta\left(X^{2}\right)}{\left(X^{2}\right)^{3 / 2}}\right)
$$

each of which has zero in the denominator on the light cone $X^{2}=0$. We will use the "finite lifetime" trick to demonstrate cancellation of divergences between two terms in the static limit. For this, first assume that the source is switched on for a finite interval of the proper time $\tau \in[a, b]$, with $a<0$ and $b>0$. On this interval one has $z^{\mu}(\tau)=[\tau, 0,0,0,0]$, so from the Eqs. (2.3) and (2.5) with account for Eq. (2.1) we obtain $\varphi(x)=\frac{g}{2} \int_{a}^{b} d \tau\left(\frac{\theta(t-\tau-r-\varepsilon)}{\left[(t-\tau)^{2}-r^{2}\right]^{3 / 2}}-\frac{\delta(t-\tau-r-\varepsilon)}{r\left[(t-\tau)^{2}-r^{2}\right]^{1 / 2}}\right)$,

where we introduced a regularizing parameter $\varepsilon>0$, shifting the singularities from the light cone. Performing an integration, one finds

$$
\varphi(t, r)=\frac{g}{2} \begin{cases}0, & t<a+r, \\ -\frac{(t-a)}{r^{2}\left[(t-a)^{2}-r^{2}\right]^{1 / 2}}, & t \in[a+r, b+r), \\ \frac{(t-b)}{r^{2}\left[(t-b)^{2}-r^{2}\right]^{1 / 2}}-\frac{(t-a)}{r^{2}\left[(t-a)^{2}-r^{2}\right]^{1 / 2}}, & t \geq b+r .\end{cases}
$$

Passing to the limit of an eternal particle worldline $a \rightarrow-\infty, b \rightarrow \infty$, one gets at any $t$ a finite result

$$
\varphi=-\frac{g}{2 r^{2}} .
$$

Similarly, divergences in the sum representation of higher $\nu$ odd-dimensional Green's functions are expected to mutually cancel and not only in the static case. We will show this explicitly for $D=3,5$.

\section{Retarded field in the wave zone}

Recall that in four dimensions the retarded electromagnetic field of a point charge consists of two parts: one proportional to $1 / r^{2}$ and representing the deformed Coulomb field, and another, acceleration dependent, which falls down as $1 / r$. The second gives a nonzero flux of the field energy-momentum (Pointing vector) through the distant sphere, and thus represents radiation. To argue that this is radiation indeed, Rohrlich [49,50] and Teitelboim [51] (see also Refs. [23,37,38,52]), computed the most long-range part of the on shell energy-momentum tensor, showing that it exhibits special properties, meaning that the corresponding part of the field energy-momentum propagates at the speed of light. Similar decomposition and reasoning holds for the gradient of the retarded scalar field of the scalar charge. All this remains valid in any spacetime dimensions, with the difference that in $D$ dimensions the area of the far sphere grows with distance as $r^{D-2}$, so the relevant asymptotic behavior of the field gradient in the wave zone is $1 / r^{D / 2-1}$. Note that in odd dimensions this power is half-integer.

In the Rohrlich-Teitelboim construction the use of certain covariantly defined quantities seems essential, so we briefly recall their definition. Consider a pointlike scalar charge moving along a worldline $z^{\mu}(\tau)$ with the $D$ velocity $v^{\mu}=d z^{\mu} / d \tau$, and denote the coordinates of the observation point as $x^{\mu}$. Consider the observation point as a top of the light cone in the past, and denote the intersection point of the light cone with the world line of a particle as $\hat{z}^{\mu}=z^{\mu}(\hat{\tau})$, where $\hat{\tau}$ is the moment of proper time corresponding to the emission of a signal propagating to the observation point at 
the speed of light. The quantity $\hat{\tau}$ is called the retarded proper time; it is determined by the equation

$$
\left(x^{\mu}-z^{\mu}(\hat{\tau})\right)^{2}=0 .
$$

In what follows, all hatted quantities will correspond to the retarded proper time $\hat{\tau}$. We then introduce two space-time vectors: a lightlike vector $\hat{R}^{\mu}=x^{\mu}-\hat{z}^{\mu}$ directed from $\hat{z}^{\mu}$ to the observation point, and a spacelike unit vector $\hat{u}^{\mu}$, orthogonal to $\hat{v}^{\mu}$. The sum of the vectors $\hat{v}^{\mu}$ and $\hat{u}^{\mu}$ forms a lightlike vector $\hat{c}^{\mu}=\hat{v}^{\mu}+\hat{u}^{\mu}$. These vectors have the following properties:

$$
\begin{array}{r}
\hat{v}^{2}=-\hat{u}^{2}=1 ; \quad \hat{c}^{2}=0 ; \quad \hat{c} \hat{v}=-\hat{c} \hat{u}=1 ; \quad \hat{v} \hat{u}=0, \\
\hat{R}^{\mu}=\hat{\rho} \hat{c}^{\mu} ; \quad \hat{\rho}=\hat{v} \hat{R} ; \quad \hat{R}^{2}=0 . \quad
\end{array}
$$

It is worth noting that $\hat{\rho}$, being the scalar product of two space-time vectors, is a Lorentz-invariant distance, equal to the distance in the Lorentz frame comoving with the charge at the retarded moment $\hat{\tau}$. Note also that for a point charge, moving along the world line $z^{\mu}(\tau)$ for an infinite proper time $-\infty<\tau<\infty$, certain care is needed to correctly define the asymptotic conditions for acceleration, for details see Ref. [51]. Here we will not discuss this subtlety, considering the simple case of periodic motion along a circle. Far from the circle, $\hat{\rho} \sim r$, so the Lorentz-invariant definition of the distance is equivalent to the naive definition. But in order to obtain Lorentz covariant expansions of tensors, one has to use $1 / \hat{\rho}$ as an expansion parameter in even dimensions and $1 / \hat{\rho}^{1 / 2}$ in odd.

Now we come back to an asymptotic structure of the on-shell energy-momentum tensor computed with the retarded solutions of the wave equation. Recall that in four-dimensional electrodynamics Teitelboim [51] demonstrated that the following decomposition holds (here we use slightly different notation):

$$
T^{\mu \nu}=T_{\text {Coul }}^{\mu \nu}+T_{\text {mix }}^{\mu \nu}+T_{\text {rad }}^{\mu \nu},
$$

where the Coulomb part falls down at infinity as $\hat{\rho}^{-4}$, the mixed part-as $\hat{\rho}^{-3}$, and the last part as $\hat{\rho}^{-2}$. Teitelboim found that in four dimensions the most long-range term of the on-shell energy-momentum tensor has the following properties:

(i) It is separately conserved $\partial_{\nu} T_{\text {rad }}^{\mu \nu}=0$.

(ii) It is proportional to the direct product of two null vectors $c^{\mu} c^{\nu}$, and therefore $c_{\mu} T_{\mathrm{rad}}^{\mu \nu}=0$.

(iii) It falls down as $1 / \hat{\rho}^{2}$ and gives positive definite energy-momentum flux through the distant sphere.

It is clear that this tensor corresponds to propagation of the field energy momentum with the speed of light. Thus, the radiation power can be computed as the flux of the energy, associated with $T_{\text {rad }}^{\mu \nu}$. Similar structure holds in the scalar theory.

Now we pass to our theory of the scalar radiation in $D$ dimensions. We substitute the retarded solution of the wave equation into the bilinear functional (2.4) and expand this quantity in inverse powers of $\hat{\rho}$. The result is as follows. The most short-range term decays as $\hat{\rho}^{4-2 D}$ in all dimensions, the mixed part is absent for $D=3$ and consists of more than one term for $D>4$, varying from $\hat{\rho}^{5-2 D}$ to $\hat{\rho}^{1-D}$, the most long-range part decays as $\hat{\rho}^{2-D}$, as expected for radiation. All the listed properties of the last term $T_{\text {rad }}^{\mu \nu}$ of this expansion hold in any $D$.

This remains true both in even and odd dimensions. An essential difference, however, is that the radiated field in the even-dimensional case depends on the particle kinematic quantities (velocity, acceleration and possibly higher derivatives of the velocity) at the retarded moment of the proper time $\hat{\tau}$ only, while in the odd case it depends on the entire history of motion before this moment. Nevertheless, due to the above properties of the on-shell stress tensor, the energy-momentum associated with the long range component of the field will propagate at the speed of light.

It is worth noting once again that the retarded field in odd dimensions, in accordance with the explicit form of the Green's functions (2.9), will have asymptotically an expansion in terms of the half-integer powers of $1 / \hat{\rho}$. A deeper discussion of the concept of asymptotic flatness in arbitrary dimensions, the definitions of null infinity by Penrose and Bondi and the Bondi-Metzner-Sachs group can be found in Refs. $[61,62]$. Half-integer powers of $1 / \hat{\rho}$ make Penrose conformal infinity ill defined in odd dimensions, while Bondi definitions are still applicable [61]. Here we will concentrate on the practical side of calculating radiation in odd dimensions, so we will not go into this interesting area.

The flux of the radiated energy-momentum passing per unit time through the $(2 \nu-1)$-dimensional sphere of radius $r$ will be given by the integral

$$
W_{2 \nu+1}^{\mu}=\int T_{\mathrm{rad}}^{\mu i} n^{i} r^{2 \nu-1} d \Omega_{2 \nu-1}, \quad i=\overline{1,2 \nu}
$$

where $d \Omega_{2 \nu-1}$ is an angular element, and $\mathbf{n}$ is a unit spacelike vector in the direction of observation.

Using this approach, we calculate scalar synchrotron radiation from a circularly moving particle in $(2+1)$ and $(4+1)$ dimensions. To verify the correctness of this calculation, we also compute the total radiation power using Fourier spectral decomposition, bearing in mind that this second method of does not depend on whether the dimension of space-time is even or odd. 


\section{Spectral decomposition}

Spectral representation can be introduced in a universal way in any dimensions, either even or odd. In terms of the Fourier transforms defined as

$$
\begin{aligned}
\varphi(x) & =\int \frac{d^{n+1} k}{(2 \pi)^{n+1}} e^{-i k x} \tilde{\varphi}(k), \\
j(x) & =\int \frac{d^{n+1} k}{(2 \pi)^{n+1}} e^{-i k x} \tilde{j}(k),
\end{aligned}
$$

where $k x=k_{\mu} x^{\mu}$ with the $(n+1)$ dimensional wave vector $k^{\mu}$, the retarded or advanced solutions of the d'Alembert equation read

$$
\tilde{\varphi}_{ \pm}(k)=\frac{\Omega \tilde{j}(k)}{k^{2} \pm i \varepsilon k^{0}}=\Omega \tilde{j}(k)\left[\frac{\mathcal{P}}{k^{2}} \mp i \pi \operatorname{sgn}\left(k^{0}\right) \delta\left(k^{2}\right)\right] .
$$

The total loss of the energy-momentum due to radiation can be presented as the work done by the current in the field presented by the half difference of the retarded and advanced solutions of the wave equation [39]:

$$
\begin{aligned}
P_{\mu} & =\frac{i}{2} \int \frac{d^{n+1} k}{(2 \pi)^{n+1}}\left[\tilde{\varphi}_{+}(k)-\tilde{\varphi}_{-}(k)\right] \tilde{j}(-k) k_{\mu} \\
& =\frac{\Omega}{(2 \pi)^{n}} \int d^{n+1} k k_{\mu}|\tilde{j}(k)|^{2} \theta\left(k^{0}\right) \delta\left(k^{2}\right) .
\end{aligned}
$$

Inserting the Fourier transform of the scalar current into the Eq. (2.19) and passing to integration over $t=\tau \gamma$ instead of $\tau$ we find the following representation for the spectralangular distribution of the total energy radiated:

$$
\frac{d P_{0}}{d \omega d \Omega}=\frac{\Omega \omega^{n-1} g^{2}}{2(2 \pi)^{n} \gamma^{2}}\left|\int_{-\infty}^{+\infty} d t \mathrm{e}^{i(\omega t-\mathbf{k z}(t))}\right|^{2}
$$

where $\omega=|\mathbf{k}|$. This generalizes the well-known $D=4$ formula [63] to arbitrary dimensions.

\section{III. $(2+1)$ THEORY}

\section{A. Coordinate representation}

The retarded solution of the Eq. (2.2) is given by Eq. (2.5):

$$
\begin{gathered}
\varphi^{\mathrm{ret}}(x)=-2 \pi \int G_{\mathrm{ret}}^{2+1}(X) j\left(x^{\prime}\right) d^{2+1} x^{\prime}, \\
G_{\mathrm{ret}}^{2+1}(X)=\frac{\theta\left(X^{0}\right)}{2 \pi} \frac{\theta\left(X^{2}\right)}{\sqrt{X^{2}}},
\end{gathered}
$$

with the current Eq. (2.3). Therefore we obtain

$$
\varphi^{\mathrm{ret}}(x)=-g \int d \tau \frac{\theta\left(X^{0}(z)\right) \theta\left(X^{2}(z)\right)}{\sqrt{X^{2}(z)}},
$$

where the vector $X^{\mu}(z)=x^{\mu}-z^{\mu}(\tau)$ joins the observation point and an instantaneous position of a particle. In fact, we need the gradient of the retarded field with respect to the observation point:

$$
\begin{aligned}
\varphi_{\mu}^{\mathrm{ret}} & \equiv \frac{\partial \varphi^{\mathrm{ret}}}{\partial x^{\mu}} \\
& =2 g \int d \tau \theta\left(X^{0}(z)\right)\left(\frac{1}{2} \frac{\theta\left(X^{2}(z)\right)}{\left(X^{2}(z)\right)^{3 / 2}}-\frac{\delta\left(X^{2}(z)\right)}{\left(X^{2}(z)\right)^{1 / 2}}\right) X_{\mu}(z) .
\end{aligned}
$$

To see the asymptotic behavior of this quantity at large distances $r \gg R_{0}$, it is convenient to present $X^{\mu}$ as

$$
X^{\mu}=x^{\mu}-z^{\mu}(\tau)=w^{\mu}+\hat{\rho} \hat{c}^{\mu}, \quad w^{\mu}=z^{\mu}(\hat{\tau})-z^{\mu}(\tau),
$$

and then expand all quantities in terms of the small ratio $w^{\mu} / \hat{\rho}$. In the leading approximation, $X^{2} \sim 2 w_{\mu} \hat{c}^{\mu}=2(w \hat{c})$. Using also the relation

$$
\theta\left(X^{0}\right) \delta\left(X^{2}\right)=\frac{\delta(\tau-\hat{\tau})}{2 \hat{\rho}},
$$

we arrive at the following expression for the leading term $\varphi_{\mu}^{\mathrm{rad}}$ of the asymptotic expansion of $\varphi_{\mu}^{\mathrm{ret}}$ in $(1 / \hat{\rho})^{1 / 2}$ :

$$
\varphi_{\mu}^{\mathrm{rad}}=\frac{g \hat{c}_{\mu}}{2^{1 / 2} \hat{\rho}^{1 / 2}} \int_{-\infty}^{\hat{\tau}} d \tau\left(\frac{1}{2(w \hat{c})^{3 / 2}}-\frac{\delta(\tau-\hat{\tau})}{(w \hat{c})^{1 / 2}}\right) .
$$

One can see that it is given by an integral over all the past worldline prior to the retarded time $\tau \leq \hat{\tau}$. This is what happens in all odd dimensions: the radiation field in the wave zone is collected from the entire history of motion preceding $\hat{\tau}$. This is similar to the tail in the radiation amplitude in four-dimensional curved space-time [44], where it is due to scattering of radiation on the space-time curvature. But, in the odd-dimensional flat space-time, the origin of tail is different. As was explained in Ref. [28], one can think of the $D$ theory as dimensionally reduced $D+1$ theory of parallel charged rods, whose projections are seen as point particles in $D$. Then, the radiation signal at infinity of the $D$ world will be collected from all pieces of rods of the $D+1$ world, in which the propagation occurs at the speed of light, but the spatial distance from the given segment of the rod to an observation point is greater (or equal at the limiting point) than its projection onto the $D$ world. Integration over the wires will produce a tail in the $D$ theory.

Each of two integrals in Eq. (3.4) diverges when $(w \hat{c})=0$. This happens in the limit $\tau \rightarrow \hat{\tau}$, where $\left(w_{\mu} \hat{c}^{\mu}\right) \rightarrow\left(u_{\mu} \hat{c}^{\mu}\right)(\hat{\tau}-\tau)=(\hat{\tau}-\tau)$. To regularize the 
integral with the delta function, we shift $\tau \rightarrow \tau+\varepsilon$ with $\varepsilon \rightarrow 0^{+}$, obtaining

$$
\int_{-\infty}^{\hat{\tau}} d \tau \frac{\delta(\tau-\hat{\tau}+\varepsilon)}{(w \hat{c})^{1 / 2}}=\frac{1}{\varepsilon^{1 / 2}}
$$

that can be rewritten as

$$
\frac{1}{\varepsilon^{1 / 2}}=\frac{1}{2} \int_{-\infty}^{\hat{\tau}-\varepsilon} \frac{d \tau}{(\hat{\tau}-\tau)^{3 / 2}}
$$

Using this representation of the delta-function term, we arrive at the finite quantity

$$
\varphi_{\mu}^{\mathrm{rad}}=\lim _{\varepsilon \rightarrow 0} \frac{g \hat{c}_{\mu}}{2^{3 / 2} \hat{\rho}^{1 / 2}} \int_{-\infty}^{\hat{\tau}-\varepsilon} d \tau\left(\frac{1}{(w \hat{c})^{3 / 2}}-\frac{1}{(\hat{\tau}-\tau)^{3 / 2}}\right),
$$

where, for brevity, we can omit the symbol $\varepsilon$ at the top point of integration, remembering that we need to perform some transformation of the integrand (usually integrating by parts) to make its finiteness manifest. It can be shown that, for the particle motion with constant velocity, the radiated part of the field gradient vanishes, as expected.

The long range energy-momentum tensor $T_{\mu \nu}^{\mathrm{rad}}$ can be found substituting the obtained expression into the bilinear form (2.4), leading to

$$
T_{\mu \nu}^{\mathrm{rad}}=\frac{g^{2} \hat{c}_{\mu} \hat{c}_{\nu}}{16 \pi \hat{\rho}} \mathcal{A}^{2}(x)
$$

where an integral radiation amplitude is introduced

$$
\mathcal{A}=\int_{-\infty}^{\hat{\tau}}\left(\frac{1}{(w \hat{c})^{3 / 2}}-\frac{1}{(\hat{\tau}-\tau)^{3 / 2}}\right)
$$

All physical information is contained in its first term, depending on the $z(\tau)$, while the second term just subtracts the divergence of the first on the upper limit. Clearly, the energy-momentum tensor obtained satisfies all the requirements of the Teitelboim definition in the $(2+1)$ dimensional space-time.

\section{B. Synchrotron radiation}

Now we proceed to calculate radiation from the circularly moving charge. The particle's worldline $z^{\mu}(\tau)$ in terms of the proper time $\tau$ will read

$$
z^{\mu}(\tau)=\left[\gamma \tau, R_{0} \cos \left(\omega_{0} \gamma \tau\right), R_{0} \sin \left(\omega_{0} \gamma \tau\right)\right],
$$

where $\gamma=E / m$ is the Lorentz factor of particle, $R_{0}$ is the radius of a circle, and $\omega_{0}$ is the frequency of rotation. The corresponding three velocity is

$$
v^{\mu}(\tau)=\gamma\left[1,-v \sin \left(\omega_{0} \gamma \tau\right), v \cos \left(\omega_{0} \gamma \tau\right)\right]
$$

where $v=R_{0} \omega_{0}$, so that $\gamma=\left(1-v^{2}\right)^{-1 / 2}$.

It will be convenient to express the retarded proper time $\hat{\tau}$ as a function of the coordinate time $t$ and the distance to the observation point from the center of the particle trajectory, which will be denoted as $R$. Using Eq. (2.13) we find

$$
\hat{\tau}=\frac{t-R}{\gamma} .
$$

Substituting this into the Eq. (2.15), we arrive at the following expressions for $\hat{\rho}$ and $\hat{c}^{\mu}$ :

$$
\begin{gathered}
\hat{\rho}=\gamma R\left(1+v \sin \left(\omega_{0} \gamma \hat{\tau}-\phi\right)\right), \\
\hat{c}^{\mu}=\frac{R}{\hat{\rho}}[1, \cos \phi, \sin \phi],
\end{gathered}
$$

where we have introduced the polar coordinates for the observation point: $x^{\mu}=[t, R \cos \phi, R \sin \phi]$. Using the parametrization of the worldline (3.8) and introducing a new angular variable and the integration parameter

$$
a=\omega_{0} \gamma \hat{\tau}-\phi+\pi / 2, \quad s=\omega_{0} \gamma(\hat{\tau}-\tau),
$$

we can present the contraction $w_{\mu} \hat{c}^{\mu}$, entering the amplitude as follows:

$$
w \hat{c}=\frac{s-v \sin a-v \sin (s-a)}{\omega_{0} \gamma(1-v \cos a)} .
$$

This gives the following representation:

$$
\mathcal{A}=\left(\omega_{0} \gamma\right)^{1 / 2} \int_{0}^{+\infty} d s\left\{\frac{(1-v \cos a)^{3 / 2}}{(s-v \sin a-v \sin (s-a))^{3 / 2}}-\frac{1}{s^{3 / 2}}\right\} .
$$

\section{The ultrarelativistic case}

It can be expected that, as in the case of synchrotron radiation in four dimensions, the radiation will be beamed and the integral amplitude can be simplified in the ultrarelativistic case $\gamma \gg 1$. Indeed, from the Eqs. (3.9) and (3.11) with account for definitions of $s$ and $a$ we can see that the main part of radiation is then formed during a small interval of proper time before the retarded time $\hat{\tau}$ (in our new notation this corresponds to small $s$ ). One can also notice that in this case radiation is beamed in the direction of the particle velocity which corresponds to $a=0$ or $\phi=\omega_{0} \gamma \hat{\tau}+\pi / 2$. This can be shown analyzing an equation for $\hat{\tau}$, similarly to an analysis in Ref. [33]. Namely, from the Eq. (2.13) we find the relation 


$$
\gamma \frac{d \hat{\tau}}{d \phi}=\frac{R_{0} \sin \left(\omega_{0} \gamma \hat{\tau}-\phi\right)}{1+v \sin \left(\omega_{0} \gamma \hat{\tau}-\phi\right)},
$$

which in the new variables reads

$$
\frac{d a}{d \phi}=-\frac{1}{1-v \cos a}
$$

This indicates that for $v \rightarrow 1$ radiation is beamed within an angle $\delta \phi \sim \delta a / \gamma^{2}$. Expanding the denominator of the first term of the integrand in Eq. (3.11) in Taylor series at $s=a=0$, we find that it has minimum of width $\delta s \sim \delta a \sim 1 / \gamma$. We can therefore find the amplitude in the leading- $\gamma$ approximation similarly to the case of the four-dimensional theory. The leading contribution to the radiation integral (3.11) will be

$$
\begin{aligned}
\mathcal{A} & =\gamma \omega_{0}^{1 / 2} \int_{0}^{+\infty} d x F(x), \\
F(x) & =\frac{1}{x^{3 / 2}}\left[\frac{\left(\hat{a}^{2}+1\right)^{3 / 2}}{\left(x^{2} / 3-\hat{a} x+\hat{a}^{2}+1\right)^{3 / 2}}-1\right],
\end{aligned}
$$

where we rescaled the variables as $x=\gamma s$ and $\hat{a}=\gamma a$. The angular distribution of the radiation power, according to general expression $(2.16)$ in $(2+1)$ dimensions will be

$$
\frac{d W_{2+1}}{d \phi}=R T_{0 i}^{\mathrm{rad}} n_{i}
$$

where the unit vector directed to the observation point is $\mathbf{n}=[\cos \phi ; \sin \phi]$. From the Eqs. (3.7), (3.9), and (3.10) we obtain the angular distribution of the radiation power

$$
\frac{d W_{2+1}}{d \phi}=\frac{g^{2} \omega_{0} \gamma \mathcal{A}^{2}}{4 \pi(1-v \cos a)^{3}} .
$$

Passing to an angular integration variable $a$ instead of $\phi$ via Eq. (3.12) and taking into account the leading- $\gamma$ behavior:

$$
\frac{d a}{d \phi} \approx-\frac{\gamma^{2}}{\hat{a}^{2}+1}
$$

we come to the leading $\gamma$ approximation for the total synchrotron scalar radiation power

$$
W_{2+1}=\frac{g^{2} \omega_{0} \gamma^{2}}{4 \pi} \int_{-\infty}^{+\infty} d \hat{a} \frac{\mathcal{A}^{2}}{\left(\hat{a}^{2}+1\right)^{2}} .
$$

The integral here is nothing but a numerical factor independent of any physical parameters.

Now we can show that the divergences in the Eq. (3.14) coming from the lower integration point in $\mathcal{A}$ mutually cancel. To demonstrate this, we have to integrate twice the first term in the integral (3.13) by parts. As a result we arrive at the following convergent integral:

$$
\begin{aligned}
\int_{0}^{+\infty} d x F(x)= & \int_{0}^{+\infty} d x \frac{x^{1 / 2}}{\left(x^{2} / 3-\hat{a} x+\hat{a}^{2}+1\right)^{5 / 2}} \\
& \times\left\{4-\frac{15(2 x / 3-\hat{a})^{2}}{x^{2} / 3-\hat{a} x+\hat{a}^{2}+1}\right\} .
\end{aligned}
$$

Numerical integration in Eq. (3.14) gives the value $4 \pi / \sqrt{3}$ up to the 5 digits for the integral in Eq. (3.14). As a result, we find the final expression for the scalar $(2+1)$ dimensional synchrotron radiation power:

$$
W_{2+1}=\frac{g^{2} \omega_{0} \gamma^{2}}{\sqrt{3}} \text {. }
$$

\section{Spectral decomposition}

To double check the validity of the above calculation, we now calculate the spectral-angular distribution of total radiated energy using the Fourier decomposition (2.20). Taking into account the beaming of radiation in the instantaneous direction of the particle's velocity and the fact that the contribution of the particle history, coming from the tail part of the Green's function is limited by the proper time interval $\delta s \sim 1 / \gamma$, we understand that the instantaneous intensity of radiation in a given direction is determined by the short arc of the circular trajectory of the order of $\delta l \sim R_{0} / \gamma$ (see, for example, Ref. [63]). In view of this we can simplify the spectral-angular distribution of radiated energy Eq. (2.20) starting with

$$
\frac{d P_{0}}{d \omega d \Omega}=\frac{\omega g^{2}}{4 \pi \gamma^{2}} \int_{-\infty}^{+\infty} d t_{1} \int_{-\infty}^{+\infty} d t_{2} e^{i \omega\left(t_{1}-t_{2}\right)-i \mathbf{k}\left(\mathbf{z}\left(t_{1}\right)-\mathbf{z}\left(t_{2}\right)\right)},
$$

where we put $n=2$ and used the formula (2.1) for $n=2$. Transforming the integration variables as

$$
t_{1}=t-t_{0} / 2, \quad t_{2}=t+t_{0} / 2,
$$

we obtain the instantaneous spectral-angular distribution of the radiation power:

$\frac{d W}{d \omega d \Omega}=\frac{d P_{0} / d t}{d \omega d \Omega}=\frac{\omega g^{2}}{4 \pi \gamma^{2}} \int_{-\infty}^{+\infty} d t_{0} e^{-i \omega t_{0}-i \mathbf{k}\left(\mathbf{z}\left(t-t_{0} / 2\right)-\mathbf{z}\left(t+t_{0} / 2\right)\right)}$.

In the ultrarelativistic case, the main contribution to this integral is given by an interval $\delta t_{0} \sim 1 / \gamma$ near $t_{0}=0$. Expanding the exponent in the Taylor series at $t_{0}=0$, we find to the leading order in $\gamma$

$$
\begin{gathered}
-i \omega t_{0}-i \mathbf{k}\left(\mathbf{z}\left(t-t_{0} / 2\right)-\mathbf{z}\left(t+t_{0} / 2\right)\right) \\
\sim-i \omega t_{0}\left(\frac{1}{2}\left(a^{2}+\frac{1}{\gamma^{2}}\right)+\frac{\omega_{0}^{2} t_{0}^{2}}{24}\right)
\end{gathered}
$$

where $\mathbf{k}=\omega[\cos \phi ; \sin \phi], \mathbf{z}(t)=R_{0}\left[\cos \omega_{0} t ; \sin \omega_{0} t\right]$ and we introduced an angular variable $a=\omega_{0} t-\phi+\pi / 2$. 
Then the leading- $\gamma$ asymptotic of the spectral distribution of radiation intensity takes the form

$$
\begin{aligned}
\frac{d W_{2+1}}{d \omega}= & \frac{\omega g^{2}}{4 \pi \gamma^{2}} \int_{0}^{2 \pi} d \phi \\
& \times \int_{-\infty}^{+\infty} d t_{0} \exp \left\{-i \omega t_{0}\left(\frac{1}{2}\left(a^{2}+1 / \gamma^{2}\right)+\frac{\omega_{0}^{2} t_{0}^{2}}{24}\right)\right\} .
\end{aligned}
$$

Similarly to the four-dimensional theory [63], after rescaling of the integration variables $\hat{a}=a \gamma$ and $t^{\prime}=\frac{1}{2}\left(\omega / \omega_{0}\right)^{1 / 3} \omega_{0} t_{0}$, one can express the integral through the Airy function [64]

$$
\operatorname{Ai}(u)=\frac{1}{2 \pi} \int_{-\infty}^{+\infty} d t^{\prime} \exp \left\{i\left(u t^{\prime}+\frac{t^{\prime 3}}{3}\right)\right\}
$$

as follows

$$
\frac{d W_{2+1}}{d \omega}=\left(\frac{\omega}{\omega_{0}}\right)^{2 / 3} \frac{g^{2}}{\gamma^{3}} \int_{-\infty}^{+\infty} d \hat{a} \mathrm{Ai}\left(\left(\frac{\omega}{\omega_{0} \gamma^{3}}\right)^{2 / 3}\left(\hat{a}^{2}+1\right)\right) .
$$

Now introduce $s=x\left(\hat{a}^{2}+1\right)$, where $x=\left(\omega / \omega_{0} \gamma^{3}\right)^{2 / 3}$, and using the formula [65]

$$
\int_{x}^{+\infty} d s \frac{\mathrm{Ai}(s)}{(s-x)^{1 / 2}}=2^{2 / 3} \pi \mathrm{Ai}^{2}\left(\frac{x}{2^{2 / 3}}\right),
$$

we perform integration in Eq. (3.18) arriving at the spectral distribution

$$
\frac{d W_{2+1}}{d \omega}=\left(\frac{\omega}{\omega_{0}}\right)^{2 / 3} \frac{g^{2}}{x^{1 / 2} \gamma^{3}} 2^{2 / 3} \pi \mathrm{Ai}^{2}\left(\frac{x}{2^{2 / 3}}\right) .
$$

We can integrate Eq. (3.19) over the spectrum passing to an integration variable $x=\left(\omega / \omega_{0} \gamma^{3}\right)^{2 / 3}$, leading to

$$
W_{2+1}=\frac{3}{2} 2^{2 / 3} \pi g^{2} \omega_{0} \gamma^{2} \int_{0}^{+\infty} d x x \operatorname{Ai}^{2}\left(\frac{x}{2^{2 / 3}}\right) .
$$

Finally, using the integral of the squared Airy function [65],

$$
\int_{0}^{+\infty} d s s \mathrm{Ai}^{2}(s)=\frac{1}{6 \sqrt{3} \pi},
$$

we obtain the total radiation power

$$
W_{2+1}=\frac{g^{2} \omega_{0} \gamma^{2}}{\sqrt{3}}
$$

coinciding with Eq. (3.15).

\section{IV. $(4+1)$ THEORY}

Calculations in the $(4+1)$-dimensional theory conceptually are the same, so we just briefly describe the main steps.

\section{A. Coordinate representation}

The retarded solution of the wave equation (2.2) reads

$$
\begin{gathered}
\varphi_{\mathrm{ret}}^{4+1}(x)=-2 \pi^{2} \int G_{\mathrm{ret}}^{4+1}\left(x-x^{\prime}\right) j\left(x^{\prime}\right) d^{4+1} x^{\prime}, \\
G_{\mathrm{ret}}^{4+1}(X)=\frac{\theta\left(X^{0}\right)}{2 \pi^{2}}\left\{\frac{\delta\left(X^{2}\right)}{\left(X^{2}\right)^{1 / 2}}-\frac{1}{2} \frac{\theta\left(X^{2}\right)}{\left(X^{2}\right)^{3 / 2}}\right\} .
\end{gathered}
$$

The corresponding field gradient takes the following form:

$$
\begin{aligned}
\varphi_{\mu}^{\mathrm{ret}}(x)= & -g \int d \tau \theta\left(X^{0}(z)\right) X_{\mu}(z)\left\{\frac{3}{2} \frac{\theta\left(X^{2}(z)\right)}{\left(X^{2}(z)\right)^{5 / 2}}\right. \\
& \left.+2 \frac{\delta^{\prime}\left(X^{2}(z)\right)}{\left(X^{2}(z)\right)^{1 / 2}}-2 \frac{\delta\left(X^{2}(z)\right)}{\left(X^{2}(z)\right)^{3 / 2}}\right\},
\end{aligned}
$$

where $\delta^{\prime}(x)=d \delta(x) / d x$. Using the relation

$$
\frac{d X^{2}(\tau)}{d \tau}=-2(v(\tau) X(z))
$$

we can integrate the $\delta^{\prime}$ term by parts, arriving at

$$
\begin{aligned}
\varphi_{\mu}^{\mathrm{ret}}(x)= & -g \int_{-\infty}^{\hat{\tau}} d \tau \frac{1}{2\left(X^{2}(z)\right)^{1 / 2}}\left\{\frac{3}{\left(X^{2}(z)\right)^{2}} X_{\mu}(z)\right. \\
& -\frac{\delta(\tau-\hat{\tau})}{\hat{\rho} X^{2}(z)} X_{\mu}(z) \\
& -\frac{\delta(\tau-\hat{\tau})}{\hat{\rho}(v(\tau) X(z))^{2}}(a(\tau) X(z)-1) X_{\mu}(z) \\
& \left.-\frac{\delta(\tau-\hat{\tau})}{\hat{\rho} v(\tau) X(z)} v_{\mu}(\tau)\right\},
\end{aligned}
$$

where we have used the relation (3.3) and introduced an acceleration five-vector $a^{\mu}(\tau)=d^{2} z^{\mu}(\tau) / d \tau^{2}$. Expanding this in terms of half-integer powers of the inverse distance $1 / \hat{\rho}$ we obtain the radiated field

$$
\begin{aligned}
\varphi_{\mu}^{\mathrm{rad}}(x)= & -\frac{g \hat{c}_{\mu}}{2^{5 / 2} \hat{\rho}^{3 / 2}} \int_{-\infty}^{\hat{\tau}} d \tau\left\{\frac{3}{2} \frac{1}{(w \hat{c})^{5 / 2}}\right. \\
& \left.-\frac{\delta(\tau-\hat{\tau})}{(w \hat{c})^{3 / 2}}-\frac{2 a \hat{c} \delta(\tau-\hat{\tau})}{(w \hat{c})^{1 / 2}(v \hat{c})^{2}}\right\} .
\end{aligned}
$$

One can simplify this expression taking into account the relations (2.14) and transformations similar to Eqs. (3.5) and (3.6): 


$$
\begin{gathered}
\int_{-\infty}^{\hat{\tau}} d \tau \frac{\delta(\tau-\hat{\tau})}{(w \hat{c})^{3 / 2}}=\frac{3}{2} \int_{-\infty}^{\hat{\tau}} \frac{d \tau}{(\hat{\tau}-\tau)^{5 / 2}}, \\
\int_{-\infty}^{\hat{\tau}} d \tau \frac{2 a \hat{c} \delta(\tau-\hat{\tau})}{(w \hat{c})^{1 / 2}(v \hat{c})^{2}}=\int_{-\infty}^{\hat{\tau}} d \tau \frac{\hat{a} \hat{c}}{(\hat{\tau}-\tau)^{3 / 2}} .
\end{gathered}
$$

This gives

$$
\begin{aligned}
\varphi_{\mu}^{\mathrm{rad}}(x)= & -\frac{g \hat{c}_{\mu}}{2^{5 / 2} \hat{\rho}^{3 / 2}} \int_{-\infty}^{\hat{\tau}} d \tau\left\{\frac{3}{2} \frac{1}{(w \hat{c})^{5 / 2}}\right. \\
& \left.-\frac{3}{2} \frac{1}{(\hat{\tau}-\tau)^{5 / 2}}-\frac{\hat{a} \hat{c}}{(\hat{\tau}-\tau)^{3 / 2}}\right\} .
\end{aligned}
$$

Now we have the sum of three integrals whose divergences at the upper limit mutually cancel: the second term is the counterterm for the first eliminating its leading divergence, while the third one is the counterterm eliminating the remaining divergence.

The $(4+1)$-dimensional on shell energy-momentum tensor evaluated with the radiated part of the field, with account for relations (2.14), will read

$$
T_{\mu \nu}^{\mathrm{rad}}(x)=\frac{g^{2} \hat{c}_{\mu} \hat{c}_{\nu}}{64 \pi^{2} \hat{\rho}^{3}} \mathcal{A}^{2}(x)
$$

where the $(4+1)$-dimensional integral radiation amplitude is

$$
\mathcal{A}(x)=\int_{-\infty}^{\hat{\tau}} d \tau\left\{\frac{3}{2} \frac{1}{(w \hat{c})^{5 / 2}}-\frac{3}{2} \frac{1}{(\hat{\tau}-\tau)^{5 / 2}}-\frac{\hat{a} \hat{c}}{(\hat{\tau}-\tau)^{3 / 2}}\right\} .
$$

Again, the energy-momentum tensor obtained satisfies all Teitelboim's requirements and therefore describes the radiated energy-momentum indeed.

\section{B. Synchrotron radiation}

Assuming the wordline to lie in the equatorial plane,

$$
z^{\mu}(\tau)=\left[\gamma \tau, R_{0} \cos \omega_{0} \gamma \tau, R_{0} \sin \omega_{0} \gamma \tau, 0,0\right]
$$

and using for the retarded proper time $\hat{\tau}=(t-R) / \gamma$, we find

$$
\begin{aligned}
\hat{\rho} & =\gamma R\left(1+v \sin \left(\omega_{0} \gamma \hat{\tau}-\phi\right) \cos \theta \cos \zeta\right), \\
\hat{c}^{\mu}= & \frac{R}{\hat{\rho}}[1, \cos \phi \cos \theta \cos \zeta, \sin \phi \cos \theta \cos \zeta, \\
& -\sin \theta \cos \zeta,-\sin \zeta],
\end{aligned}
$$

where we used the hyperspherical coordinates for the observation point

$$
\begin{aligned}
x^{\mu}= & {[t, R \cos \phi \cos \theta \cos \zeta, R \sin \phi \cos \theta \cos \zeta,} \\
& -R \sin \theta \cos \zeta,-R \sin \zeta] .
\end{aligned}
$$

Calculating the contractions $w \hat{c}$ and $\hat{a} \hat{c}$, we then pass to the integration variable $s=\omega_{0} \gamma(\hat{\tau}-\tau)$ and introduce the angular variable $a=\omega_{0} \gamma \hat{\tau}-\phi+\frac{\pi}{2}$. Zero values of the angular variables correspond to the particle's instant direction of motion at the retarded moment of proper time $\hat{\tau}$. After some algebra, we arrive at the following representation of the integral amplitude of radiation:

$$
\begin{aligned}
\mathcal{A}(x)= & \left(\omega_{0} \gamma\right)^{3 / 2} \int_{0}^{+\infty} d s G(s), \\
G(s)= & \frac{3}{2}\left(\left[\frac{\delta \Delta}{s-v \Delta(\sin a+\sin (s-a))}\right]^{5 / 2}-\frac{1}{s^{5 / 2}}\right) \\
& -\frac{v \Delta \sin a}{s^{3 / 2}(1-v \Delta \cos a)},
\end{aligned}
$$

where we denoted $\Delta=\cos \theta \cos \zeta$ and $\delta=1-v \cos a$. One can see that in the ultrarelativistic case $v \rightarrow 1$ the main part of the energy is radiated in a narrow cone around the instantaneous direction of velocity $a=0, \theta=0, \zeta=0$ within $\delta a \sim 1 / \gamma, \delta \theta \sim 1 / \gamma, \delta \zeta \sim 1 / \gamma$. Using the leading- $\gamma$ relation between the angular variables following from the equation for $\hat{\tau}(2.13)$,

$$
\frac{d a}{d \phi}=-\frac{1}{1-v \Delta \cos a} \approx-\frac{1}{2 \gamma^{2}}\left(\hat{a}^{2}+\hat{\theta}^{2}+\hat{\zeta}^{2}+1\right),
$$

and rescaling the integration variables $\{s, a, \theta, \zeta\} \rightarrow$ $\{x=s \gamma, \hat{a}=a \gamma, \hat{\theta}=\theta \gamma, \hat{\zeta}=\zeta \gamma\}$, we obtain from the Eq. (4.5) in the leading order:

$G(x)=\frac{\gamma^{5 / 2}}{x^{3 / 2}}\left\{\frac{3}{2 x}\left(\left[\frac{\hat{A}}{x^{2} / 3-\hat{a} x+\hat{A}}\right]^{5 / 2}-1\right)-\frac{2 \hat{a}}{\hat{A}}\right\}$,

where we denoted $\hat{A}=\hat{a}^{2}+\hat{\theta}^{2}+\hat{\zeta}^{2}+1$.

Divergences in the different terms of the radiation integral (4.6) at $s \sim 0$ at the upper limit are canceled after the triple integration by parts of the first term in the integrand. Doing these integrations one can use different regularization parameters $\varepsilon=+0$ absorbing in them some numerical factors.

The total radiation power now will be given by the integral

$$
W_{4+1}=\int d \phi d \theta d \zeta R^{3} \sin \theta \sin ^{2} \zeta T_{0 i}^{\mathrm{rad}} n_{i}
$$

After triple integration by parts in the radiation amplitude, the angular distribution of the radiation power will read 
$\frac{d W_{4+1}}{d \hat{a} d \hat{\theta} d \hat{\zeta}}=\frac{g^{2} \omega_{0}^{3} \gamma^{6}}{4 \pi^{2}} \hat{A}\left\{\int_{0}^{+\infty} d x \frac{315 x^{1 / 2} \Phi}{2 \Lambda^{9 / 2}}\left(\frac{\Phi^{2}}{\Lambda}-\frac{140}{315}\right)\right\}^{2}$

where we denoted $\Phi=2 x / 3-\hat{a}$ and $\Lambda=x^{2} / 3-\hat{a} x+\hat{A}$. In the leading- $\gamma$ approximation, the angular distribution is beamed, this is used similarly to the previous section. Integration over the angles is relegated to the Appendix. The resulting factor is obtained by numerical integration after some analytic transformations and has the value $1 / \sqrt{27}$ up to 5 digits. The total radiation power of the $(4+1)$-dimensional scalar synchrotron radiation will read

$$
W_{4+1}=\frac{g^{2} \omega_{0}^{3} \gamma^{6}}{\sqrt{27}}
$$

\section{Spectral decomposition}

Now from Eq. (2.20) we get

$$
\frac{d P_{0}}{d \omega d \Omega}=\frac{\omega^{3} g^{2}}{16 \pi^{2} \gamma^{2}} \int_{-\infty}^{+\infty} d t_{1} \int_{-\infty}^{+\infty} d t_{2} e^{i \omega\left(t_{1}-t_{2}\right)-i \mathbf{k}\left(\mathbf{z}\left(t_{1}\right)-\mathbf{z}\left(t_{2}\right)\right)}
$$

After the transformation (3.16), we present the radiation power as

$$
\begin{aligned}
\frac{d W}{d \omega d \Omega} & =\frac{d P_{0} / d t}{d \omega d \Omega} \\
& =\frac{\omega^{3} g^{2}}{16 \pi^{2} \gamma^{2}} \int_{-\infty}^{+\infty} d t_{0} e^{-i \omega t_{0}-\mathbf{k}\left(\mathbf{z}\left(t-t_{0} / 2\right)-\mathbf{z}\left(t+t_{0} / 2\right)\right)} .
\end{aligned}
$$

The main contribution to the integral comes from the region $\delta t_{0} \sim 1 / \gamma$ near $t_{0}=0$. Thus one can expand an expression in the exponent of the integrand into Taylor series at $t_{0}=0$ up to the leading order in $\gamma$ as

$$
\begin{aligned}
- & i \omega t_{0}-i \mathbf{k}\left(\mathbf{z}\left(t-t_{0} / 2\right)-\mathbf{z}\left(t+t_{0} / 2\right)\right) \\
= & -\frac{i \omega t_{0}}{2 \gamma^{2}}\left(\hat{a}^{2}+\hat{\theta}^{2}+\hat{\zeta}^{2}+1+\frac{\omega_{0}^{2} t_{0}^{2} \gamma^{2}}{12}\right),
\end{aligned}
$$

where the angular variables are chosen in accordance with the wave zone calculations. We also rescaled the angular variables multiplying on $\gamma$ to stretch the limits $\hat{a}, \hat{\theta}$, $\hat{\zeta} \in(-\infty,+\infty)$. After that we arrive at the spectral distribution of the radiation power in the form

$$
\frac{d W_{4+1}}{d \omega}=\frac{\omega^{3} g^{2}}{16 \pi^{2} \gamma^{5}} \int_{\mathbb{R}^{3}} d \hat{a} d \hat{\theta} d \hat{\zeta} \int_{-\infty}^{+\infty} d t_{0} \exp \left\{-\frac{i \omega t_{0}}{2 \gamma^{2}}\left(1+\hat{a}^{2}+\hat{\theta}^{2}+\hat{\zeta}^{2}+\frac{\omega_{0}^{2} t_{0}^{2} \gamma^{2}}{12}\right)\right\}
$$

Using the spherical coordinates for integration over the rescaled angular variables

$$
\hat{a}=\rho \cos \alpha \sin \beta, \quad \hat{\theta}=\rho \sin \alpha \sin \beta, \quad \hat{\zeta}=\rho \cos \beta,
$$

where $\rho \in[0 ;+\infty), \alpha \in[0 ; 2 \pi)$ and $\beta \in[0 ; \pi]$, we find the following integral representation of the spectral distribution of radiation intensity

$$
\begin{aligned}
\frac{d W_{4+1}}{d \omega}= & \frac{\omega^{3} g^{2}}{4 \pi \gamma^{5}} \int_{0}^{+\infty} d \rho \rho^{2} \\
& \times \int_{-\infty}^{+\infty} d t_{0} \exp \left\{-\frac{i \omega t_{0}}{2 \gamma^{2}}\left(1+\rho^{2}+\frac{\omega_{0}^{2} t_{0}^{2} \gamma^{2}}{12}\right)\right\} .
\end{aligned}
$$

Integration over $t_{0}$ could be performed in terms of the Airy function (3.17)

$$
\frac{d W_{4+1}}{d \omega}=\frac{\omega^{3} g^{2}}{\omega_{0} \gamma^{5}}\left(\frac{\omega_{0}}{\omega}\right)^{1 / 3} \int_{0}^{+\infty} d \rho \rho^{2} \operatorname{Ai}\left(\left(\frac{\omega}{\gamma^{3} \omega_{0}}\right)^{2 / 3}\left(\rho^{2}+1\right)\right) .
$$

We can rescale an integration variable $r=\rho\left(\omega / \omega_{0} \gamma^{3}\right)^{1 / 3}$ and introduce a dimensionless parameter $x=\left(\omega / \omega_{0} \gamma^{3}\right)^{2 / 3}$ to simplify this to

$$
\frac{d W_{4+1}}{d \omega}=\left(\frac{\omega_{0}}{\omega}\right)^{1 / 3} \frac{\omega^{2} g^{2}}{\gamma^{2}} \int_{0}^{+\infty} d r r^{2} \operatorname{Ai}\left(r^{2}+x\right) .
$$

Integration over $r$ can be performed using the integral [65]

$$
\begin{aligned}
\int_{0}^{+\infty} d r r^{2} \operatorname{Ai}\left(r^{2}+x\right)= & \frac{\pi}{2^{2 / 3}}\left(\operatorname{Ai}^{\prime}\left(x / 2^{2 / 3}\right)\right)^{2} \\
& -\frac{\pi x}{2^{4 / 3}} \operatorname{Ai}^{2}\left(x / 2^{2 / 3}\right) .
\end{aligned}
$$

Passing to new dimensionless parameter $s=\left(\omega / 2 \omega_{0} \gamma^{3}\right)^{2 / 3}$ we finally obtain

$$
\frac{d W_{4+1}}{d \omega}=2 \pi g^{2} \gamma^{3} \omega_{0}^{2} s^{5 / 2}\left(\left(\mathrm{Ai}^{\prime}(s)\right)^{2}-s \mathrm{Ai}^{2}(s)\right) .
$$

The total power of synchrotron radiation in $(4+1)$ dimensions will read

$$
W_{4+1}=6 \pi g^{2} \omega_{0}^{3} \gamma^{6} \int_{0}^{+\infty} d s s^{3}\left(\left(\operatorname{Ai}^{\prime}(s)\right)^{2}-s \mathrm{Ai}^{2}(s)\right) .
$$

Using the integrals [65]

$$
\begin{aligned}
\int_{0}^{+\infty} d s s^{3}\left(\mathrm{Ai}^{\prime}(s)\right)^{2} & =\frac{5}{18 \sqrt{3} \pi}, \\
\int_{0}^{+\infty} d s s^{4} \mathrm{Ai}^{2}(s) & =\frac{2}{9 \sqrt{3} \pi},
\end{aligned}
$$


we find for the total power

$$
W_{4+1}=\frac{g^{2} \omega_{0}^{3} \gamma^{6}}{\sqrt{27}}
$$

which coincides with the result of calculation in the wave zone Eq. (4.7).

\section{Arbitrary D}

Combining Eq. (3.15) with Eq. (4.8) and with the known result for $D=4$ [66], we can conjecture that the power of the scalar synchrotron radiation in $D=n+1$ dimensions is given by the following formula:

$$
W_{n+1}^{\mathrm{sc}}=g^{2}\left(\frac{\omega_{0} \gamma^{2}}{\sqrt{3}}\right)^{n-1} \text {. }
$$

\section{CONCLUSION}

In this article, we showed that despite the violation of the Huygens principle in odd dimensions, radiation from an accelerated charge can be calculated by integrating the energy-momentum flux in the asymptotic wave zone in a standard way. However, an important difference with the even-dimensional case is that the distant component of the gradient of the retarded field depends on the entire history of motion preceding the retarded proper time. Another unusual feature is that the amplitude is initially represented by the sum of divergent integrals, whose divergences are mutually canceled. This is due to the nature of the odddimensional Green's functions, which should be defined as regularized distributions. Integrating by parts, you can make the cancellation explicit and get the final result.

We explicitly calculated the synchrotron radiation power in the dimensions $D=3,5$ showing that the integral over history (tail) is effectively localized in the ultrarelativistic limit, so the obtained expression can be found analytically. In addition, we found that, together with the previously known result for $D=4$ [66], the total radiation power is described by the universal formula (4.9). We conjecture that this might be valid in any $D$.

Our results were double checked by the method of spectral decomposition, based on expansion of all quantities into Fourier integrals. This second method is closer to the calculations of quantum theory, and it does not distinguish between even and odd dimensions. Note that the spectral-angular distributions have similar features in the ultrarelativistic case (beaming) in both odd and even dimensions.

Now we briefly discuss the relationship to other works. Our formula agrees with the calculation of Shuryak et al. [33] of the synchrotron radiation power in $2+1$ dimensions by means of the radiation reaction, but disagrees with their result in $D=5$. We also disagree with the results of
Yaremko obtained through the radiation reaction. We agree with the qualitative estimates of the power of Mironov et al. [67] and with the results obtained by Cardoso et al. [26] for even-dimensional space-time.

\section{ACKNOWLEDGMENTS}

The authors are grateful to the referee for the valuable suggestions that were included in the revised version of the article. We thank Boris Kosyakov and Sunny Vagnozzi for references and remarks. This work of M. Kh. was supported by the "BASIS" Foundation Grant No. 18-2-6-41-1. The authors would like to acknowledge the networking support of the COST Action No. CA16104.

\section{APPENDIX: TOTAL RADIATION POWER IN 4 + 1}

The computation below belongs to Bogush [68]. Consider the integral

$$
\begin{aligned}
J & =\frac{1}{4 \pi^{2}} \int_{\mathbb{R}^{3}} d \hat{a} d \hat{\theta} d \hat{\zeta}(\hat{A}+1) I^{2}, \\
I & =\int_{0}^{+\infty} d x \sqrt{x} \frac{f^{\prime}}{f^{9 / 2}}\left(\frac{315}{2} \frac{f^{\prime 2}}{f}-70\right), \\
f(x) & =x^{2} / 3-a x+\hat{A}+1,
\end{aligned}
$$

where $\hat{A}=\hat{a}^{2}+\hat{\theta}^{2}+\hat{\zeta}^{2}$. Introducing cylindrical coordinates in the space of $(\hat{a}, \hat{\theta}, \hat{\zeta})$ by use of $b^{2}=\hat{\theta}^{2}+\hat{\zeta}^{2}$, we obtain

$$
\begin{aligned}
J & =\frac{1}{2 \pi} \int_{-\infty}^{+\infty} d \hat{a} \int_{0}^{+\infty} d b b\left(\hat{a}^{2}+b^{2}+1\right) I^{2}, \\
f(x) & =\frac{3}{4}(2 x / 3-\hat{a})^{2}+\frac{1}{4} \hat{a}^{2}+b^{2}+1 .
\end{aligned}
$$

Passing to the integration variable $y=2 x / 3-\hat{a}$ and denoting $c^{2}=\hat{a}^{2}+4\left(b^{2}+1\right)$, we find

$$
\begin{aligned}
f(x) & =\frac{1}{4}\left(3 y^{2}+c^{2}\right), \quad f^{\prime}(x)=y, \\
I & =70 \times 2^{9}\left(\frac{3}{2}\right)^{3 / 2} \int_{-\hat{a}}^{+\infty} d y \frac{y \sqrt{y+\hat{a}}}{\left(3 y^{2}+c^{2}\right)^{9 / 2}}\left(\frac{9 y^{2}}{3 y^{2}+c^{2}}-1\right) .
\end{aligned}
$$

This integral can be evaluated in terms of the complete elliptic integrals $E(d)$ and $K(d)$ 


$$
\begin{aligned}
I & =\frac{1}{4 \times 3^{1 / 4} C}(A(2 E(d)+K(d))+B K(d)), \\
A & =-288 \hat{a}\left(b^{2}+1\right)\left(\hat{a}^{2}+3 b^{2}+3\right), \\
B & =\sqrt{3} \sqrt{\hat{a}^{2}+b^{2}+1}\left(28 \hat{a}^{4}+44 \hat{a}^{2}\left(b^{2}+1\right)-80\left(b^{2}+1\right)^{2}\right), \\
C & =\left(\hat{a}^{2}+4\left(b^{2}+1\right)\right)^{2}\left(\hat{a}^{2}+b^{2}+1\right)^{15 / 4}, \\
d & =\frac{1}{2}\left(\frac{\sqrt{3} \hat{a}}{2 \sqrt{\hat{a}^{2}+b^{2}+1}}+1\right) .
\end{aligned}
$$

As a result, we obtain the amplitude of radiation intensity in the form of double integral

$$
J=\frac{1}{32 \sqrt{3} \pi} \int_{-\infty}^{+\infty} d \hat{a} \int_{0}^{+\infty} d b \frac{b(A(2 E(d)+K(d))+B K(d))^{2}}{\left(\hat{a}^{2}+4\left(b^{2}+1\right)\right)^{4}\left(\hat{a}^{2}+b^{2}+1\right)^{13 / 2}}
$$

which can be easily evaluated numerically, giving $J=1 / \sqrt{27}$ up to 5 digits.

[1] V. A. Rubakov, Large and infinite extra dimensions: An introduction, Usp. Fiz. Nauk 171, 913 (2001) [Phys. Usp. 44, 871 (2001)].

[2] I. Ya Aref'eva, Holographic approach to quark-gluon plasma in heavy ion collisions, Phys. Usp. 57, 527 (2014).

[3] V. Cardoso, R. Emparan, D. Mateos, P. Pani, and J. V. Rocha, Holographic collisions in confining theories, J. High Energy Phys. 01 (2014) 138.

[4] P. Ehrenfest, How do the fundamental laws of physics make manifest that space has 3 dimensions?, Ann. Phys. (N.Y.) 366, 440 (1920).

[5] R. Courant and D. Hilbert, Methods of Mathematical Physics: Partial Differential Equations, Wiley Classics Library (Wiley, New York, 2008).

[6] J. Hadamard, Lectures on Cauchy's Problem in Linear Partial Differential Equations (Dover Publications, New York, 2014).

[7] D. Ivanenko and A. Sokolov, Sov. Phys. Dokl. 36, 37 (1940); Classical Field Theory (State Publishing House of Technical and Theoretical Literature, Moscow, 1951) (in Russian) [Klassische Feldtheorie (Akademie-Verlag, Berlin, 1953)].

[8] A. O. Barvinsky and S. N. Solodukhin, Echoing the extra dimension, Nucl. Phys. B675, 159 (2003).

[9] C. Deffayet and K. Menou, Probing gravity with spacetime Sirens, Astrophys. J. 668, L143 (2007).

[10] D. Andriot and G. L. Gómez, Signatures of extra dimensions in gravitational waves, J. Cosmol. Astrophys. Phys. 06 (2017) 048; Erratum, J. Cosmol. Astrophys. Phys. 05 (2019) E01.

[11] K. Chakravarti, S. Chakraborty, K. S. Phukon, S. Bose, and S. SenGupta, Constraining extra-spatial dimensions with observations of GW170817, Classical Quantum Gravity, https://doi.org/10.1088/1361-6382/ab8355.
[12] L. Visinelli, N. Bolis, and S. Vagnozzi, Brane-world extra dimensions in light of GW170817, Phys. Rev. D 97, 064039 (2018).

[13] H. Yu, Z. C. Lin, and Y.X. Liu, Gravitational waves and extra dimensions: A short review, Commun. Theor. Phys. 71, 991 (2019).

[14] O. K. Kwon, S. Lee, and D. D. Tolla, Gravitational waves as a probe of the extra dimension, Phys. Rev. D 100, 084050 (2019).

[15] V. Cardoso, L. Gualtieri, and C. J. Moore, Gravitational waves and higher dimensions: Love numbers and KaluzaKlein excitations, Phys. Rev. D 100, 124037 (2019).

[16] S. Vagnozzi and L. Visinelli, Hunting for extra dimensions in the shadow of M87*, Phys. Rev. D 100, 024020 (2019).

[17] D. Gal'tsov, P. Spirin, and T. N. Tomaras, Gravitational bremsstrahlung in ultra-Planckian collisions, J. High Energy Phys. 01 (2013) 087.

[18] E. Berti, V. Cardoso, and B. Kipapa, Up to eleven: Radiation from particles with arbitrary energy falling into higher-dimensional black holes, Phys. Rev. D 83, 084018 (2011).

[19] D. V. Galtsov, G. Kofinas, P. Spirin, and T. N. Tomaras, Classical ultrarelativistic bremsstrahlung in extra dimensions, J. High Energy Phys. 05 (2010) 055.

[20] V. Frolov, M. Snajdr, and D. Stojkovic, Interaction of a brane with a moving bulk black hole, Phys. Rev. D 68, 044002 (2003).

[21] D. Gal'tsov, E. Melkumova, and P. Spirin, Branestrahlung: Radiation in the particle-brane collision, Phys. Rev. D 93, 045018 (2016).

[22] D. Gal'tsov, E. Melkumova, and P. Spirin, Piercing of domain walls: New mechanism of gravitational radiation, J. High Energy Phys. 01 (2018) 120.

[23] B. P. Kosyakov, Exact solutions of classical electrodynamics and the Yang-Mills-Wong theory in even-dimensional space-time, Theor. Math. Phys. 119, 493 (1999). 
[24] B. P. Kosyakov, Radiation in electrodynamics and in YangMills theory, Phys. Usp. 35, 135 (1992).

[25] V. Cardoso, O. J. C. Dias, and J. P. S. Lemos, Gravitational radiation in D-dimensional space-times, Phys. Rev. D 67, 064026 (2003).

[26] V. Cardoso, M. Cavaglia, and J. Q. Guo, Gravitational Larmor formula in higher dimensions, Phys. Rev. D 75, 084020 (2007).

[27] M. Gurses and O. Sarioglu, Lienard-Wiechert potentials in even dimensions, J. Math. Phys. (N.Y.) 44, 4672 (2003).

[28] D. V. Galtsov, Radiation reaction in various dimensions, Phys. Rev. D 66, 025016 (2002).

[29] P. O. Kazinski, S. L. Lyakhovich, and A. A. Sharapov, Radiation reaction and renormalization in classical electrodynamics of point particle in any dimension, Phys. Rev. D 66, 025017 (2002).

[30] P. O. Kazinski and A. A. Sharapov, Radiation back-reaction and renormalization in classical field theory with singular sources, Theor. Math. Phys. 143, 798 (2005).

[31] Y. Yaremko, Radiation reaction in $2+1$ electrodynamics, J. Math. Phys. (N. Y.) 48, 092901 (2007).

[32] Y. Yaremko, Self-force in $2+1$ electrodynamics, J. Phys. A 40, 13161 (2007).

[33] E. Shuryak, H.-U. Yee, and I. Zahed, Self-force and synchrotron radiation in odd space-time dimensions, Phys. Rev. D 85, 104007 (2012).

[34] Y. Yaremko, Renormalization and radiation reaction in 2+ 1 electrodynamics, J. Phys. Stud. 15, 1003 (2011).

[35] D.-C. Dai and D. Stojkovic, Origin of the tail in Green's functions in odd-dimensional space-times, Eur. Phys. J. Plus 128, 122 (2013).

[36] A. I. Harte, P. Taylor, and É. É. Flanagan, Foundations of the self-force problem in arbitrary dimensions, Phys. Rev. D 97, 124053 (2018).

[37] B. P. Kosyakov, Self-interaction in classical gauge theories and gravitation, Phys. Rep. 812, 1 (2019).

[38] B. P. Kosyakov, Introduction to the Classical Theory of Particles and Fields (Springer, Berlin, Heidelberg, 2007).

[39] P. A. M. Dirac, Classical theory of radiating electrons, Proc. R. Soc. A 167, 148 (1938).

[40] G. E. Shilov, Generalized Functions and Partial Differential Equations (Gordon and Breach, New York, 1968).

[41] V. V. Nesterenko, A. Feoli, and G. Scarpetta, Dynamics of relativistic particle with Lagrangian dependent on acceleration, J. Math. Phys. (N.Y.) 36, 5552 (1995).

[42] B. P. Kosyakov and V. V. Nesterenko, Stability of Zitterbewegung of a rigid particle, Phys. Lett. B 384, 70 (1996).

[43] R. A. Anpilogov, Stable compact motions of a particle driven by a central force in six-dimensional spacetime, Ann. Phys. (Amsterdam) 366, 133 (2016).

[44] B. S. DeWitt and R. W. Brehme, Radiation damping in a gravitational field, Ann. Phys. (N.Y.) 9, 220 (1960).

[45] L. Barack and A. Pound, Self-force and radiation reaction in general relativity, Rep. Prog. Phys. 82, 016904 (2019).
[46] D. V. Gal'tsov and P. A. Spirin, Radiation reaction in curved even-dimensional spacetime, Gravitation Cosmol. 13, 241 (2007).

[47] P. A. Spirin, Massless field emission in the space-time of extra dimensions, Gravitation Cosmol. 15, 82 (2009).

[48] L. D. Landau and E. M. Lifshitz, The Classical Theory of Fields (Elsevier, New York, 2013).

[49] F. Rohrlich, The definition of electromagnetic radiation, Nuovo Cimento (1955-1965) 21, 811 (1961).

[50] F. Rohrlich, Classical Charged Particles (World Scientific, Singapore, 2007).

[51] C. Teitelboim, Splitting of the Maxwell tensor: Radiation reaction without advanced fields, Phys. Rev. D 1, 1572 (1970).

[52] D. V. Gal'tsov and P. Spirin, Radiation reaction reexamined: Bound momentum and Schott term, Gravitation Cosmol. 12, 1 (2006).

[53] D. V. Gal'tsov, Radiation reaction and energy-momentum conservation, Fundam. Theor. Phys. 162, 367 (2011).

[54] O. Birnholtz and S. Hadar, Gravitational radiation-reaction in arbitrary dimension, Phys. Rev. D 91, 124065 (2015).

[55] O. Birnholtz and S. Hadar, Action for reaction in general dimension, Phys. Rev. D 89, 045003 (2014).

[56] R. A. Porto, The effective field theorist's approach to gravitational dynamics, Phys. Rep. 633, 1 (2016).

[57] R. A. Breuer, P. L. Chrzanowksi, H. G. Hughes, and C. W. Misner, Geodesic synchrotron radiation, Phys. Rev. D 8, 4309 (1973).

[58] R. R. Landim, On the Laplace equation in d-dimension, arXiv:0901.3488.

[59] D. Zwillinger, Table of Integrals, Series, and Products (Elsevier Science, New York, 2014).

[60] G. N. Watson, A Treatise on the Theory of Bessel Functions, Cambridge Mathematical Library (Cambridge University Press, Cambridge, England, 1995).

[61] K. Tanabe, S. Kinoshita, and T. Shiromizu, Asymptotic flatness at null infinity in arbitrary dimension, Phys. Rev. D 84, 044055 (2011).

[62] M. Henneaux and C. Troessaert, The asymptotic structure of electromagnetism in higher spacetime dimensionss, Phys. Rev. D 99, 125006 (2019).

[63] V. N. Baier, V. M. Katkov, and V.S. Fadin, Radiation of Relativistic Electrons (Atomizdat, Moscow, 1973) (in Russian).

[64] M. Abramowitz and I. A. Stegun, Handbook of Mathematical Functions with Formulas, Graphs, and Mathematical Tables (Dover, New York, 1964).

[65] V. Olivier and S. Manuel, Airy Functions And Applications To Physics, 2nd ed. (World Scientific, Singapore, 2010).

[66] A. O. Barut and D. Villarroel, Radiation reaction and mass renormalization in scalar and tensor fields and linearized gravitation, J. Phys. A 8, 156 (1975).

[67] A. Mironov and A. Morozov, Is strong gravitational radiation predicted by TeV-Gravity? JETP Lett. 85, 6 (2007).

[68] I. Bogush (private communication). 